\title{
Experimental results of the laserwire emittance scanner for LINAC4 at CERN ${ }^{2}$
}

\author{
Thomas Hofmann ${ }^{\mathrm{a}, \mathrm{b}, \mathrm{c}, *}$, Gary E. Boorman ${ }^{\mathrm{b}}$, Alessio Bosco ${ }^{\mathrm{b}}$, Enrico Bravin ${ }^{\mathrm{a}}$, \\ Stephen M. Gibson ${ }^{\mathrm{b}}$, Konstantin O. Kruchinin ${ }^{\mathrm{b}}$, Uli Raich ${ }^{\mathrm{a}}$, Federico Roncarolo ${ }^{\mathrm{a}}$, \\ Francesca Zocca ${ }^{a}$ \\ ${ }^{a}$ CERN, Geneva 1211, Switzerland \\ ${ }^{\mathrm{b}}$ John Adams Institute at Royal Holloway, University of London, Egham TW20 OEX, United Kingdom \\ ${ }^{\text {c }}$ Friedrich-Alexander-University, Erlangen 91054, Germany
}

\section{A R T I C L E I N F O}

Available online 16 February 2016

Keywords:

Laserwire

Linac

Emittance

$\mathrm{H}^{-}$

Diamond

Fibre optics

\begin{abstract}
A B S T R A C T
Within the framework of the LHC Injector Upgrade (LIU), the new LINAC4 is currently being commissioned to replace the existing LINAC2 proton source at CERN. After the expected completion at the end of 2016, the LINAC4 will accelerate $\mathrm{H}^{-}$ions to $160 \mathrm{MeV}$. To measure the transverse emittance of the $\mathrm{H}^{-}$ beam, a method based on photo-detachment is proposed. This system will operate using a pulsed laser with light delivered via an optical fibre and subsequently focused onto the $\mathrm{H}^{-}$beam. The laser photons have sufficient energy to detach the outer electron and create $\mathrm{H}^{0} / \mathrm{e}^{-}$pairs. In a downstream dipole, the created $\mathrm{H}^{0}$ particles are separated from the unstripped $\mathrm{H}^{-}$ions and their distribution is measured with a dedicated detector. By scanning the focused laser beam across the $\mathrm{H}^{-}$beam, the transverse emittance of the $\mathrm{H}^{-}$beam can be reconstructed. This paper will first discuss the concept, design and simulations of the laser emittance scanner and then present results from a prototype system used during the $12 \mathrm{MeV}$ commissioning of the LINAC4.
\end{abstract}

(c) 2016 Elsevier B.V. All rights reserved.

\section{Introduction}

The photo-detachment process for hydrogen ions has been known since 1959 [1]. The advent of $\mathrm{H}^{-}$ion based linear accelerators (linacs) made possible to apply this process in order to measure parameters of the $\mathrm{H}^{-}$beam [2]. For today's modern high current linacs this technique offers numerous benefits. Due to its non-invasive nature, beam parameters can be measured parasitically and since no mechanical parts are needed to intercept the particle beam, the risk of overheated and damaged components is eliminated. Since the reduction of downtimes is a major aim for any accelerator, this non-destructive instrument can contribute to maximise the efficiency.

The advantages are particularly relevant for the LINAC4 which is due to deliver high brightness beams reliably to the LHC injector complex. The declared aim of the HL-LHC, to increase the integrated luminosity by a factor of ten [3], is directly dependent on the LINAC4 performance and reliability. The increased final energy of $160 \mathrm{MeV}$ creates further challenges for the beam instrumentation. Due to the

\footnotetext{
International Conference on Laser Applications at Accelerators, LA3NET 2015.

* Corresponding author at: CERN, Geneva 1211, Switzerland. Tel.: +41 227677130.

E-mail address: thomas.hofmann@cern.ch (T. Hofmann).
}

long range of protons at this energy (e.g. $>20 \mathrm{~cm}$ in graphite) conventional methods such as slits or scrapers cannot be used. By using a laser as a 'slit', firstly the range problem is solved and secondly the laser can be adjusted such that just a tiny fraction of $\mathrm{H}^{-}$ions become neutralised $\mathrm{H}^{\circ}$. This makes the laserwire principle truly non-invasive. A comprehensive overview of the laserwire system and the integration at LINAC4 can be found at $[4,5]$.

In this paper the emphasis is put on measurement results obtained while commissioning such a laserwire system on a $12 \mathrm{MeV} \mathrm{H}^{-}$beam. Since the principle of this technique has been already demonstrated in other facilities [6-8], the focus was on making advances in key parameters of the system. In this regard a low-power laser was used which made it possible to design a simple fibre-based system, which runs very reliably and has imperceptible impact on the ion beam.

\section{Instrument design}

\subsection{LINAC4}

LINAC4 is the first step in the LHC Injector Upgrade (LIU) program which is essential for delivering the high brightness beams for the HL-LHC upgrade. After determining the principal machine 
Table 1

Principal LINAC4 parameters.

\begin{tabular}{ll}
\hline Parameter & Value \\
\hline Overall linac length & $90 \mathrm{~m}$ \\
Output energy & $160 \mathrm{MeV}$ \\
Bunch frequency & $352 \mathrm{MHz}$ \\
Beam pulse length & $400 \mu \mathrm{s}$ \\
Beam pulse repetition rate & $0.83 \mathrm{~Hz}$ \\
Average pulse current & $40 \mathrm{~mA}$ \\
Nominal transverse emittance & $0.4 \pi \mathrm{mm}$ mrad \\
\hline
\end{tabular}

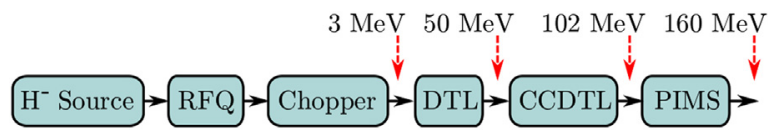

Fig. 1. LINAC4 block diagram. After generating the ion beam in a caesiated surface source the radiofrequency quadrupole (RFQ) first accelerates and then generates a bunched beam at an energy of $3 \mathrm{MeV}$. The chopper selects a temporal slice of the beam for further acceleration. DTL, CCDTL (Cell-Coupled) and PIMS (Pi-ModeStructure) are the drift tubes linacs (DTL) required to accelerate the beam to the final energy of $160 \mathrm{MeV}$.

parameters in a technical design report in 2006 [9], the civil engineering was completed and the machine is currently in the commissioning phase. Table 1 and Fig. 1 provide an overview on the machine's essential parameters.

The determination of the transverse emittance at the end of the linac is essential in order to avoid high losses when the beam is injected into the PS-Booster [10]. Therefore the $160 \mathrm{MeV}$ region is the envisaged final location for the proposed laserwire instrument. To advance with the instrument's design while the commissioning of LINAC4 is still underway, the phases at $3 \mathrm{MeV}$ and $12 \mathrm{MeV}$ (after the first DTL tank) were used to test the laserwire prototype.

\subsection{Instrument concept}

Due to the small binding energy of the outer electron of $0.75 \mathrm{eV}$, the $\mathrm{H}^{-}$ion can be neutralised rather easily. Collisions with residual gas atoms [4] or strong magnetic fields in the Tesla range can detach one electron. The principle of our instrument is based on electron detachment due to collisions with photons. This process has a large cross-section of $>3 \times 10^{-17} \mathrm{~cm}^{2}$ in the range between $500 \mathrm{~nm}$ and $1200 \mathrm{~nm}$ photon wavelength [1].

To sample the transverse phase space of an ion beam, a common method is to select thin slices of the beam and measure its angular distribution, known as the slit/grid method [11]. In analogy to this principle, Fig. 2 shows a schematic of the laserwire, which in contrast to the slit/grid method, is non-destructive.

\subsection{Laser system}

Since the laser system was described in detail in previous publications $[4,5]$ it will be summarised here. The general parameters of the chosen working point of the laser source are listed in Table 2. The low pulse energy in comparison to other laserwire systems makes it possible to deliver the laser light to the particle beam via a long optical fibre ( $10 \mathrm{~m}$ was already achieved during the experiments completed at 3 and $12 \mathrm{MeV}$ and a longer fibre will be used for the final system). This greatly reduces the complexity of the overall laser light delivery system.

The laser beam is kept at a fixed position and focused into the vacuum vessel with a diameter of approximately $150 \mu \mathrm{m}$. Due to the quasi-monomode beam quality $\left(M^{2}=1.8\right)$, the laser beam diameter remains almost constant when colliding with the millimetre-size particle beam. The vertical scanning of the laser

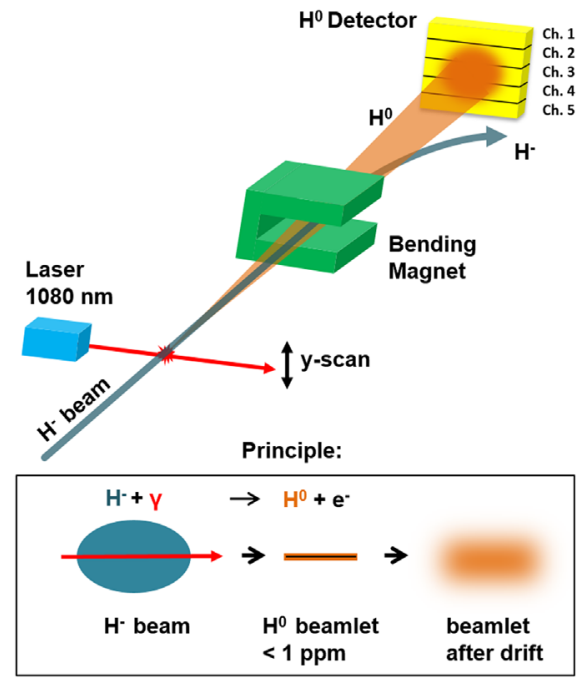

Fig. 2. Concept of the laserwire emittance measurement. A focused laser beam crosses the $\mathrm{H}^{-}$beam and detaches electrons from the ions. The resulting neutral $\mathrm{H}^{0}$ atoms drift unperturbed towards a detector while the $\mathrm{H}^{-}$ions are deflected in a downstream bending magnet. By measuring the $\mathrm{H}^{0}$ profiles in the detection plane, the angular distribution of the beam is gained. A scan of the laser through the $\mathrm{H}^{-}$ beam allows sampling the transverse phase space [12].

Table 2

Laser parameters.

\begin{tabular}{ll}
\hline Parameter & Value \\
\hline Wavelength & $1080 \mathrm{~nm}$ \\
Pulse energy & $154 \mu \mathrm{J}$ \\
Pulse length (FWHM) & $80 \mathrm{~ns}$ \\
Pulse frequency & $60 \mathrm{kHz}$ \\
$M^{2}$ & 1.8 \\
\hline
\end{tabular}

beam is performed by a remote controlled stage. A CCD camera and a fast photodiode are used to monitor the laser beam quality.

\section{4. $H^{0}$ detection system}

For the measurements at $12 \mathrm{MeV}$, the $20 \mathrm{~mm} \times 20 \mathrm{~mm}$ polycrystalline diamond detector with 5 strip channels already exploited during the $3 \mathrm{MeV}$ experiments [13] was used to detect the neutralised $\mathrm{H}^{0}$ atoms. Contrary to the $3 \mathrm{MeV}$ case, the $12 \mathrm{MeV}$ $\mathrm{H}^{0}$ atoms fully traverse the $500 \mu \mathrm{m}$ thick diamond substrate. This eliminates problems with piled up charge inside the diamond and greatly increases the signal level. A simulation of the ionisation inside the diamond was performed using the software package SRIM [14]. It was found that one $\mathrm{H}^{0}$ loses $9.3 \mathrm{MeV}$ when traversing the diamond. The charge collected by the detector can be calculated as:

$Q_{\text {Diamond }}=n_{\mathrm{H}^{0}} \cdot e \cdot \frac{E_{\text {ionize }}}{E_{\text {Gen }}} \cdot C C E$

where $n_{\mathrm{H}^{0}}$ is the number of $\mathrm{H}^{0}$ hitting the diamond, $E_{\text {ionize }}$ the energy that one $\mathrm{H}^{0}$ loses in the diamond and $E_{\mathrm{Gen}}$ the average energy to generate an electron-hole pair, which is $13.1 \mathrm{eV}$ for diamond [15]. Depending on the diamond material quality the Charge Collection Efficiency (CCE) can vary significantly. For the detector used in the measurements a CCE between 10\% and $20 \%$ was assumed [16]. Using this value, a simulation was executed to estimate the collected charge taking into account the power of the laser as presented in Table 2, the beam dynamics and the size of a detector strip channel $(3.5 \mathrm{~mm} \times 18 \mathrm{~mm})$. The simulation result 


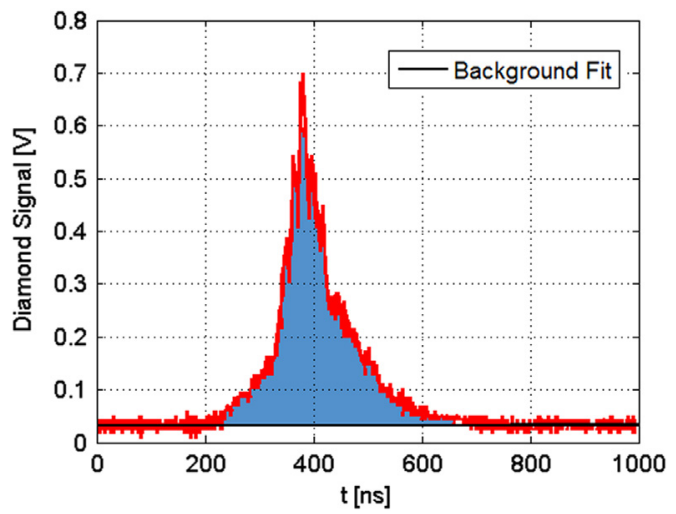

Fig. 3. Red: Signal on diamond in response to one laser pulse (see Fig. 8) and constant $\mathrm{H}^{0}$ background; Hatching: integrated charge.

was a charge yield of max. $2 \times 10^{-9} \mathrm{C}$, generated by one laser pulse in one detector channel.

Such a charge creates a voltage in the order of $1 \mathrm{~V}$ (across a $50 \Omega$ termination) for the duration of a laser pulse, and the signal can therefore be digitised without pre-amplification.

\section{System characterisation}

\subsection{Signal amplitude at diamond detector}

The signals received from the diamond detector were compared to simulations to verify that the laser and detector systems worked as expected. The plot of Fig. 3 shows the signal from the diamond detector caused by one laser pulse. The background level is a result of $\mathrm{H}^{0}$ generated by interactions with residual gas atoms [4]. To determine the charge created by the laser interaction, this background was subtracted and the resulting distribution was then integrated (see hatching in Fig. 3).

For this resulting charge, values up to $1.2 \times 10^{-9} \mathrm{C}$ have been measured depending on the laser and detector position with respect to the $\mathrm{H}^{-}$beam. This is nearly a factor of 2 lower than expected, but taking into account the uncertainties in the simulation due to the uncertain CCE value of the detector, the agreement can be considered as acceptable. It must be also noted that the CCE changes due to radiation damage and therefore the amount of charge collected also varied during the two month measurement campaign.

\subsection{Signal linearity along beam pulse}

In order to determine the change of the CCE of the diamond detector response during the ion beam pulse, a comparison with a beam current transformer (BCT) was performed. Special attention was paid to this test, since the previous results using the $3 \mathrm{MeV}$ beam [13] showed rather non-linear behaviour.

The signal from the BCT situated immediately after the laser interaction point (IP) was measured for the duration of the phase space scan. The signal from the diamond detector was recorded as shown in Fig. 4. A whole phase space scan was made for each detector channel. The phase space was then integrated for comparison with the averaged BCT signal during the scan period, as shown in Fig. 5.

The plot shows that the behaviour of each of the three diamond detector channels is very similar. The agreement between each channel and the averaged BCT signal is within $10 \%$ for each sample of the pulse. The residual disagreement is the result of a small number of particles bypassing the diamond detector. This is a

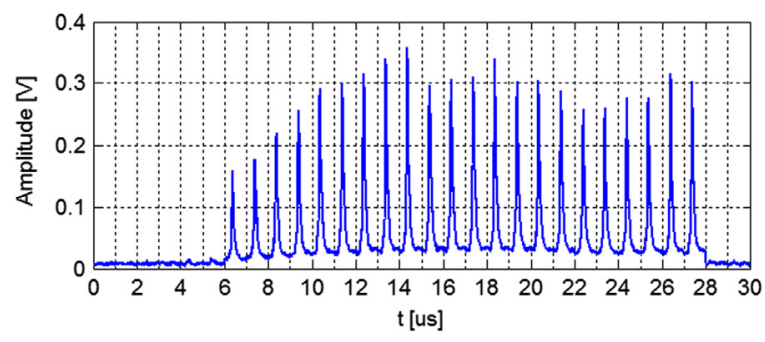

Fig. 4. Diamond signal segments during the $400 \mu \mathrm{s} \mathrm{H}^{-}$ion pulse. The laser was pulsed at $60 \mathrm{kHz}$ and creates 24 signal peaks in the signal of the diamond detector. For each peak, a $1 \mu$ s time interval was recorded. The plot shows these signal intervals stitched together for the duration of the ion pulse.

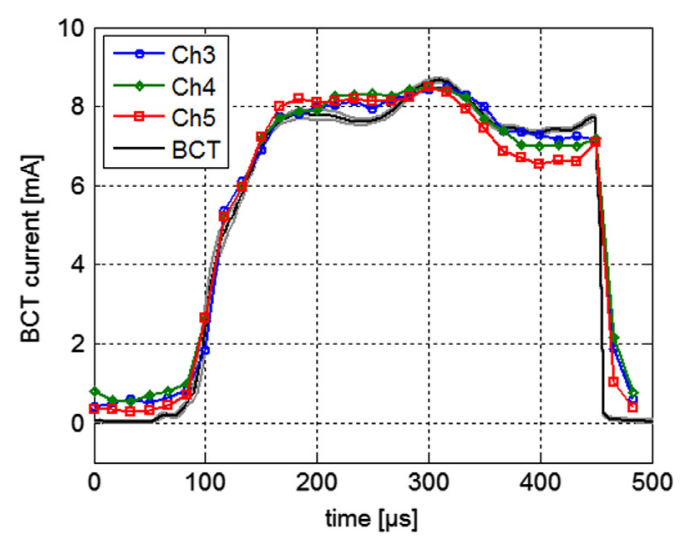

Fig. 5. Comparison of the averaged beam current with the signals of 3 channels of the diamond detector. The diamond signals were normalised to compare with the BCT signal.

significant improvement over the $3 \mathrm{MeV}$ measurements, for which the diamond signal dropped dramatically in the second half of the beam pulse, which was caused by the pile-up of implanted $\mathrm{H}^{-}$ ions in the diamond bulk material.

\subsection{Homogeneity of diamond detector}

The signals from each of the diamond channels were compared to check the homogeneity of the polycrystalline diamond material. Since the DAQ system was still in development, the system was only capable of recording the signals from three out of the five detector channels. The focused laser beam was kept at a fixed position, and the detector was moved to record each detector channel at the same position in phase space.

The curves in Fig. 6 correspond to the integrated charge per laser pulse in each segment. The difference between channels is well within their error bars, which correspond to the standard deviation (SD) of successive recordings. The increase in error during the rising and falling edge is due to the missing synchronization of the $60 \mathrm{kHz}$ laser with the ion beam, which introduced a $17 \mu$ s jitter on the signal from the diamond detector.

\subsection{Signal linearity versus laser pulse energy}

A key aspect in determining the beam profile and the emittance is the linearity of the diamond detector with respect to the impinging particles. It is difficult to change the LINAC4 ion beam current without changing the beam divergence and size, therefore a different approach was chosen. Assuming a linear relation between the laser pulse energy and the number of stripped $\mathrm{H}^{-}$ ions, the linearity of the diamond detector can be determined by varying the laser power. 


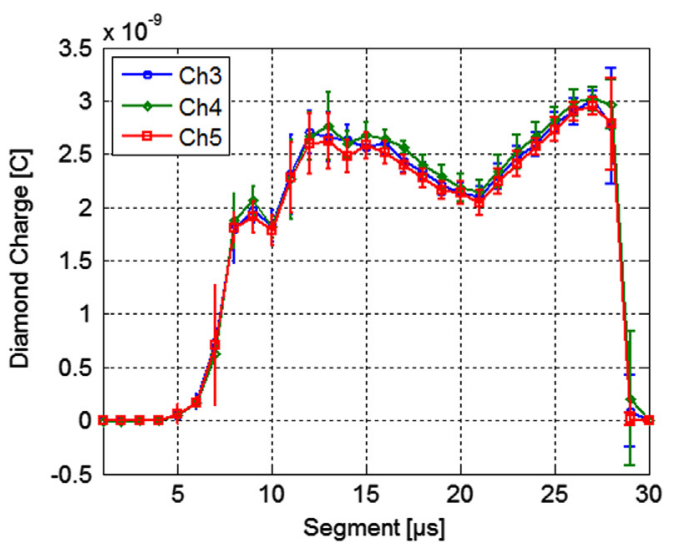

Fig. 6. Signal charge of three detector channels, which were recorded with laser light at fixed position and each detector channel moved to the same location.

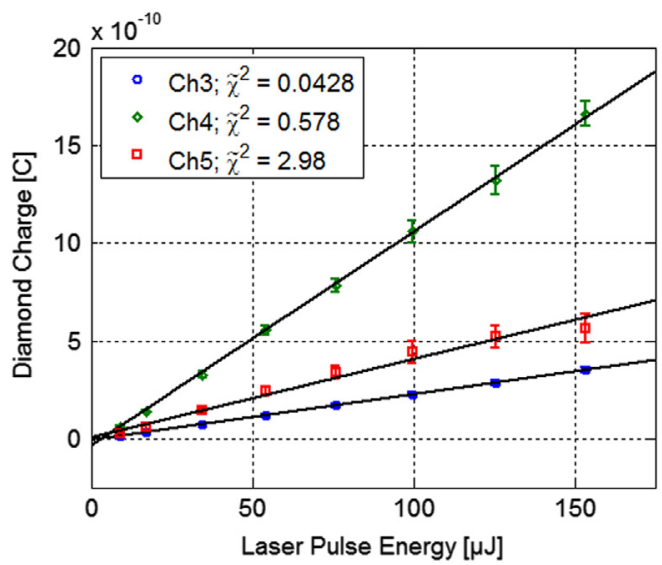

Fig. 7. Integrated charge recorded by the diamond detector channels as a function of the laser pulse energy. Solid lines: Linear fit for each channel.

The laser pulse energy at the end of the fibre was measured and the laser focus was positioned at the centre of the $\mathrm{H}^{-}$beam. The results with the detector at a fixed position are shown in Fig. 7. The values of the slopes are different due to the different positions of the detector channels. However, the linear fit indicates that the signal distortion due to non-linearities is within the error bars of the signal (see $\tilde{\chi}^{2}$ values).

\section{Data analysis and results}

\subsection{Temporal beam properties}

The measurement campaign was mainly focused on sampling the phase space and then reconstructing the transverse beam emittance and profile. However, by using a high bandwidth detection system temporal beam properties could also be measured. Fig. 8 shows the laser pulse recorded before delivery to the beam pipe and the signal from the diamond detector. The similarity of the shapes shows clearly that the signal from the diamond detector originates from $\mathrm{H}^{0}$ atoms created by the laser pulse. The delay of the 2 signals of 194 ns (SD, 4 ns) has three contributions: the time of the laser delivery to the IP with the particle beam (51 ns); the time-of-flight (TOF) of the particle from IP to the diamond detector (71 ns); and 72 ns delay in the cable from the diamond detector to the DAQ system. This system cannot replace a dedicated system for TOF-measurement but it gives a hint regarding the energy of the $\mathrm{H}^{-}$ions.

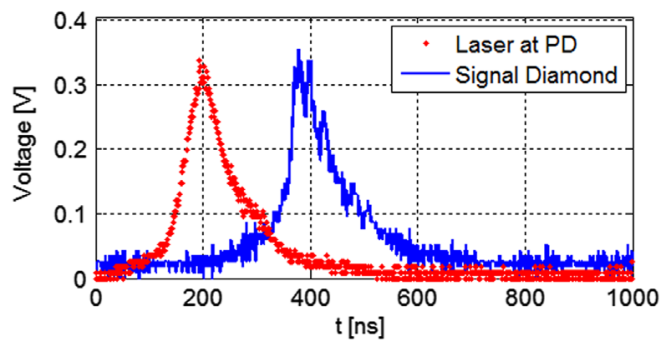

Fig. 8. Laser pulse recorded with fast photodiode (PD) and signal of diamond detector.

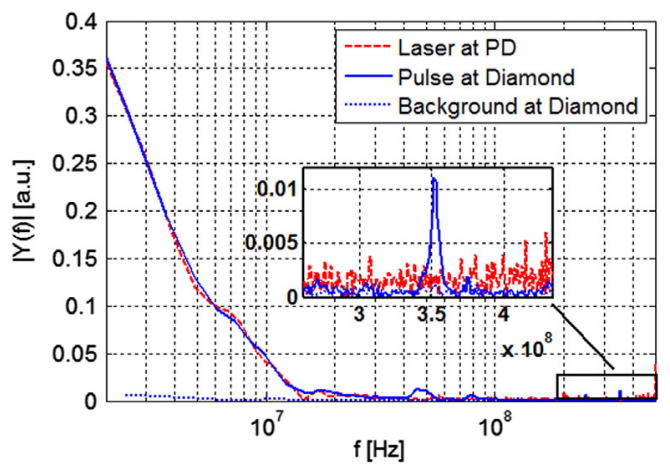

Fig. 9. Fourier transformation of the laser pulse at the PD, the pulse at the diamond detector and the constant background arriving at the diamond detector. Zoom: Peak at $352 \mathrm{MHz}$ bunch freq.

A further observation was made by performing a FourierTransform of the diamond detector signal. In Fig. 9 the spectra of the signals shown in Fig. 8 are plotted. The frequency components with high amplitude in the lower frequency range are very similar when comparing the laser pulse recorded at the PD and the diamond. In the high-frequency range a clear signal from the diamond is recorded at exactly $352 \mathrm{MHz}$, the bunch frequency of the LINAC4. This illustrates the potential of the diamond detector regarding measurements with highest time resolution.

The absence of the $352 \mathrm{MHz}$ peak in the background signal is also well understood. The $\mathrm{H}^{0}$ particles in the background are generated by collisions with residual gas atoms all along the accelerator, each with different kinetic energies and the arriving particles are therefore expected to be completely de-bunched.

\subsection{Transverse phase space and beam profile}

As for the $3 \mathrm{MeV}$ case, a system for sampling only the vertical phase space was developed for the $12 \mathrm{MeV}$ experiments.

Just before the set of emittance measurements started, a fault in the LINAC4 machine interlock system allowed a full $\mathrm{H}^{-}$pulse to hit the diamond detector. The resulting radiation damage left only one diamond channel surviving with sufficient CCE. Consequently the emittance scan consisted of vertically scanning the detector through the whole $\mathrm{H}^{0}$ beamlet in steps of $1.8 \mathrm{~mm}$. The detector scan was repeated for each laser position, changed in steps of $0.5 \mathrm{~mm}$.

The raw data (of the kind shown in Fig. 4) was analysed in three steps. The detector signal was first low-pass filtered to remove high frequency noise. Then a linear fit to the background level was made (see Fig. 3) and subtracted from the signal, before summing the charge of the resulting signal. The obtained values in vertical phase space are shown in Fig. 11.

To evaluate the precision of the novel laserwire instrument, the beam was sampled also with a conventional slit/grid instrument. 


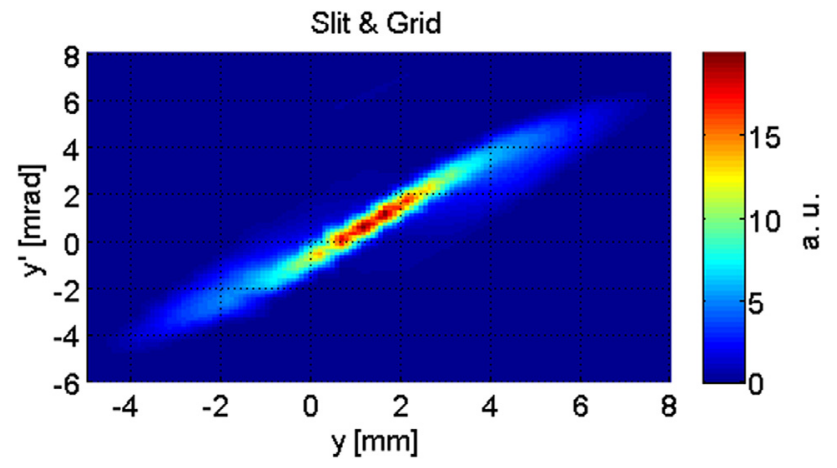

Fig. 10. Phase space sampled with the slit/grid method.

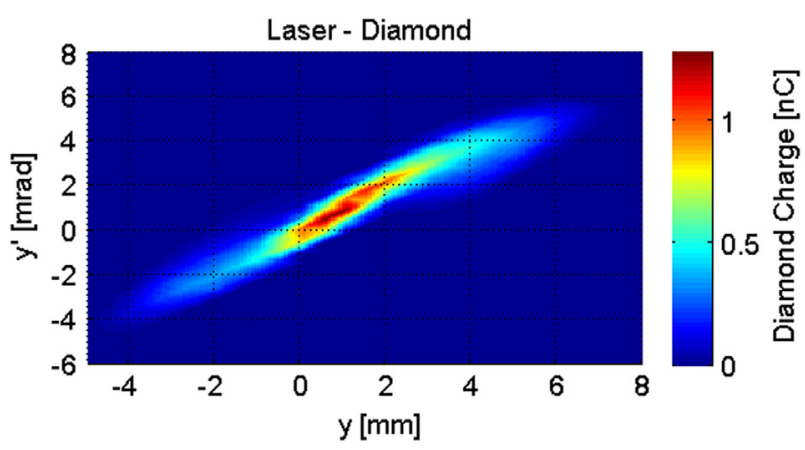

Fig. 11. Phase space sampled with the laser/diamond system.

Fig. 10 shows the result of the reference measurement using the same $\mathrm{H}^{-}$beam parameters.

Comparison of both phase space distributions shows a remarkable overall agreement. Minor differences are mainly present in the angular distribution. The measurement using the laserwire exhibits a slightly broadened angular profile, which is caused by the lower spatial resolution of the diamond detector.

Integrating the phase space in the divergence domain, the resulting beam profile is plotted for both instruments in Fig. 12. The disagreement in terms of profile shape and therefore extracted beam size is well below $2 \%$. The error bars, calculated as the signal's amplitude variation in time along the LINAC4 pulse, are in the same range for both the laser/diamond and slit/grid systems.

\subsection{Emittance reconstruction}

The value of the normalised emittance can be expressed as follows:

$\varepsilon_{R M S, \text { norm }}=\beta \gamma \sqrt{\left\langle y^{2}\right\rangle\left\langle y^{\prime 2}\right\rangle-\left\langle y y^{\prime}\right\rangle^{2}}$

here $y$ and $y^{\prime}$ represent the amount of arriving particles at a given vertical position and divergence angle. The relativistic $\beta$ and $\gamma$ factors allow relating the geometric to the normalised emittance.

The measurement depends strongly on the sampled phase space area due to the constant noise level of a real instrument. To suppress the noise a simple method is commonly used to exclude all values in phase space which are below a certain threshold corresponding to a percentage of the maximum amplitude $[17,18]$. Fig. 13 shows the resulting emittance values, measured with the laser/diamond instrument and the slit/grid reference system as a function of the applied threshold. The characteristic kink in this curve marks the spot where the noise is largely suppressed and the sampled signal starts to originate from impinging particles. For the laserwire system this point is quite distinct at $1 \pm 0.1 \%$. The

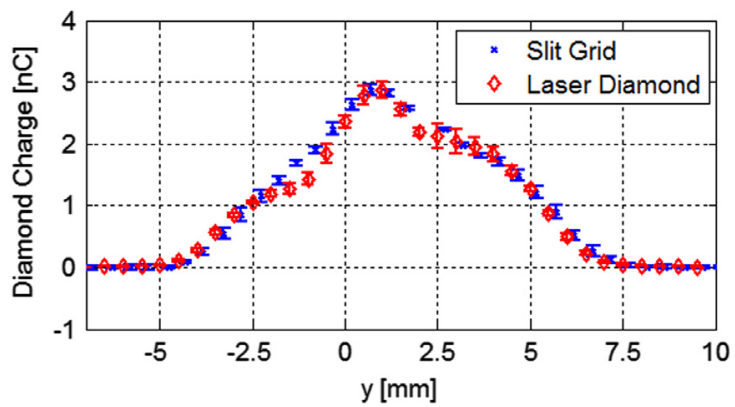

Fig. 12. Comparison of the measured beam profiles: Signal of the slit/grid signal normalised to the charge measured with the diamond detector.

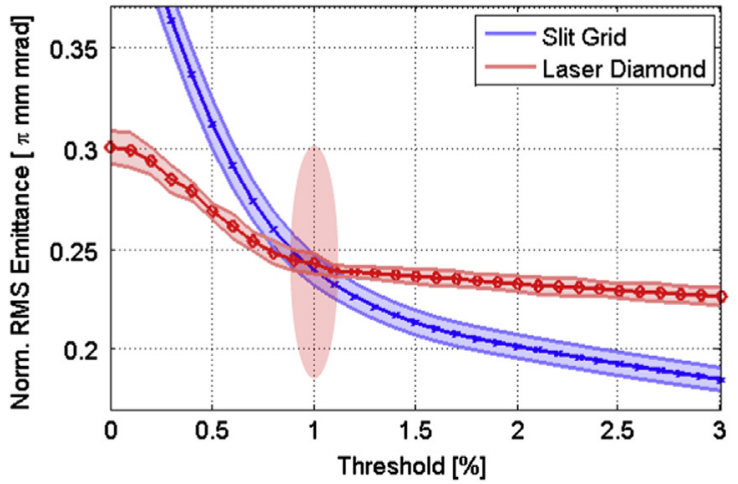

Fig. 13. Normalised emittance value resulting from both instruments. Dependence on used threshold for noise suppression.

Table 3

Comparison of emittance result values.

\begin{tabular}{lll}
\hline System & Slit/grid & Laser/diamond \\
\hline Norm. RMS emittance $[\pi \mathrm{mm}$ mrad] & 0.240 & 0.243 \\
Threshold dependence & \pm 0.0084 & \pm 0.0024 \\
SD during LINAC4 pulse & \pm 0.0070 & \pm 0.0026 \\
\hline
\end{tabular}

equivalent position for the slit/grid is not so clearly defined but is in the same region. Assuming the same threshold of $1 \%$ for both systems the resulting emittance values are summarized in Table 3 including the uncertainty due to the choice of a reasonable threshold (between $0.9 \%$ and $1.1 \%$ ) and the standard deviation (SD) of measurements during the LINAC4 pulse.

\section{Summary}

An initial prototype design of this laser-based instrument was first demonstrated during the $3 \mathrm{MeV}$ commissioning stage of Linac4 [4,5]. During the $12 \mathrm{MeV}$ commissioning the design of instrument was refined and its performance was characterized in depth.

The amplitude of the diamond detector signal was compared with prior simulations, where an acceptable agreement was found. The linearity of the detector was characterized in the time domain along the LINAC4 pulse and also in comparison to the number of impinging particles. In both domains the response of the detector was linear. The homogeneity of the different detector channels also proved to be satisfactory. Overall the diamond detector system exhibited a significantly improved performance due to the higher range of the impinging particles compared with the previous $3 \mathrm{MeV}$ tests. 
With regard to the laser system, a noteworthy change in comparison to the $3 \mathrm{MeV}$ setup is the extension of the fibre delivery of the laser beam to the IP to $10 \mathrm{~m}$. The transported laser pulses did not show any distortion and the transmission efficiency was above $70 \%$ even for pulses with peak power above $2 \mathrm{~kW}$.

Analysing the measurements with the laserwire system allowed to determine temporal beam parameters as the time-offlight or the bunch structure but the main focus of the measurements remained the profile and emittance reconstruction.

The results of the emittance measurements with the laserwire system were compared with the well-established slit/grid method. Vertical phase space plots and beam profile projections agree very well, however the resulting RMS emittance value is dependent on the threshold for noise suppression. Given a reasonable choice of threshold (red area in Fig. $13-0.9 \% . .1 .1 \%$ ), the emittance results of the two systems agree to within $3 \%$.

\subsection{Outlook}

The next steps of the LINAC4 commissioning at $50 \mathrm{MeV}$ and $100 \mathrm{MeV}$ will be used to test a modified version of the instrument, to measure the detached electrons and reconstruct the beam profile in a non-invasive manner [19]. Tests are ongoing to further increase the fibre delivery length of the laser beam in order to install the laser-head in a radiation safe environment outside the accelerator tunnel.

The encouraging results of the presented measurement campaign at $12 \mathrm{MeV}$ represent a milestone on the way to the final instrument, which will be used at the $160 \mathrm{MeV}$ LINAC4 beam. In preparation for its permanent operation, the electrode design of the diamond detector and its data acquisition readout chain will be re-designed to provide higher angular resolution and faster emittance measurements. The laser system will need to be modified to be able to scan in both the horizontal and vertical planes.

\section{Acknowledgements}

This paper is dedicated to the memory of our late colleague Christoph Gabor, a fruitful and enthusiastic scientist. We are grateful to the LINAC4 operation team and our colleagues in the
CERN profile measurement section for the strong support during this project. Furthermore we want to thank the FETS project team for lending us their laser source and especially Alan Letchford and Jürgen Pozimski for the fruitful discussions. We acknowledge support from the STFS FETS grant and from the Marie Curie Networks LA ${ }^{3}$ NET and oPAC which are funded by the European Commission under Grant agreement number 289191 and 289485.

\section{References}

[1] S.J. Smith, D.S. Burch, Physics Review 116 (5) (1959).

[2] W.B. Cottingame, et al., IEEE Transactions Nuclear Science NS32 (1985).

[3] CERN, LHC/HL-LHC plan, 〈http://hilumilhc.web.cern.ch/about/hl-lhc-project〉, 2014 (accessed 17.02.15).

[4] T. Hofmann, et al., Physical Reviews Accelerators and Beams 18 (2015) 122801.

[5] S.M. Gibson et al., A fibre coupled, lowpower laserwire emittance scanner at CERN LINAC4, in: Proceedings of IPAC14, Dresden, Germany.

[6] Y. Liu, et al., Nuclear Instruments and Methods in Physics Research Section A 612 (2010) 241

[7] T. Tomisawa et al., Investigation of photo neutralization efficiency of high intensity $\mathrm{H}$ - beam with ND:YAG laser In J-PARC, in: Proceedings of DIPAC 2005, Lyon, France.

[8] R. Connolly et al., A laser-wire beam-energy and beam-profile monitor at the BNL LINAC, MOP194, In: Proceedings of 2011 PAC, New York, NY, USA

[9] F. Gerigk, M. Vretenar et al., Linac4 technical design report. Technical report, CERN, 2006, CERN AB 2006084 ABP/RF.

[10] W. Weterings et al., Status of the $160 \mathrm{MeV} \mathrm{H}^{-}$injection into the CERN PSB, TUPPR091, In: Proceedings of IPAC2012, New Orleans, Louisiana, USA.

[11] F. Zocca et al., Profile and emittance measurements at the CERN LINAC4 $3 \mathrm{MeV}$ test stand, In: Proceedings of IBIC13, Oxford, UK.

[12] T. Hofmann et al., Laser based stripping system for measurements of the transverse emittance of $\mathrm{H}^{-}$beam at the CERN LINAC4 MOPME075, In: Proceedings of IPAC2013, Shanghai, China.

[13] T. Hofmann et al., Status of and future plans for the CERN LINAC4 emittance meter based on laser electron-detachment and a diamond strip-detector, In: Proceedings of IBIC14, Monterey, CA, USA.

[14] J.F. Ziegler, et al., Nuclear Instruments and Methods in Physics Research Section A 268 (2010) 1818-1823.

[15] R.C. Alig, S. Bloom, C.W. Struck, Physical Review B 22 (12) (1980) 5565.

[16] E. Grießmayer, personal communication, 2013.

[17] B. Cheymol et al., First results from beam measurements at the $3 \mathrm{MeV}$ test stand for CERN LINAC4, In: Proceedings of DIPAC2011, Hamburg, Germany.

[18] G. Bellodi et al., LINAC4 $45 \mathrm{keV}$ proton beam measurements, In: Proceedings of LINAC2012, Tel-Aviv, Israel.

[19] T. Hofmann et al., Design of a laser-based profile monitor for LINAC4 commissioning at $50 \mathrm{MeV}$ and $100 \mathrm{MeV}$, In: Proceedings of IBIC15, Melbourne, Australia. 